\title{
Susan Friedlander's Contributions in Mathematical Fluid Dynamics
}

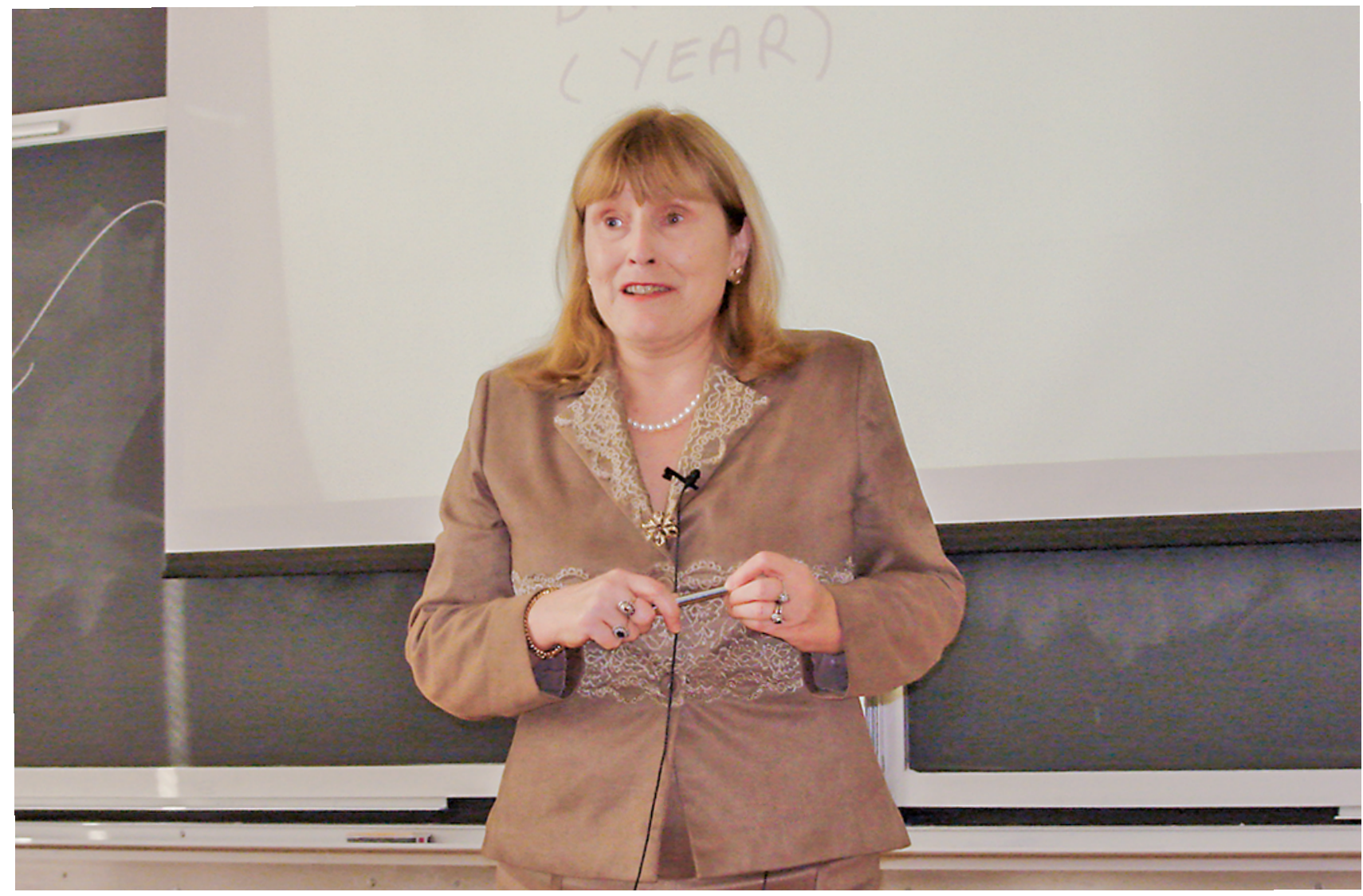

\section{Alexey Cheskidov, Nathan Glatt-Holtz, Natasa Pavlovic, Roman Shvydkoy, and Vlad Vicol}

Alexey Cheskidov is a professor of mathematics at the University of Illinois at Chicago. His email address is acheskid@uic.edu.

Nathan Glatt-Holtz is an assistant professor of mathematics at Tulane University. His email address is negh@tu 7 ane. edu.

Natasa Pavlovic is a professor of mathematics at the University of Texas Austin. Her email address is natasa@math. utexas. edu.

Roman Shvydkoy is a professor of mathematics at the University of Illinois at Chicago. His email address is shvydkoy@uic. edu.

Vlad Vicol is a professor of mathematics at New York University. His email address is vicol@cims.nyu.edu.

Opening photo is of Susan Friedlander lecturing at the Fields Institute in 2008.
Susan Friedlander received her undergraduate degree in mathematics from University College London in 1967. Having been awarded one of the prestigious Kennedy Scholarships to study at the Massachusetts Institute of Technology, Friedlander moved to the US and earned her MS degree at MIT in 1970. She subsequently started her

Communicated by Notices Associate Editor Daniela De Silva.

For permission to reprint this article, please contact: reprint-permission@ams.org.

DOI: https://doi.org/10.1090/noti2237 
PhD studies at Princeton University, completing her doctorate thesis under the title "Spin Down in a Rotating Stratified Fluid" in 1972 with the supervision of the fluid dynamicist Louis Norberg Howard. After being a visiting member at New York University's Courant Institute of Mathematical Sciences, Friedlander moved to the University of Illinois at Chicago, where she worked as a Professor until 2008. Since then, Friedlander is a Professor and the Director of the Center for Applied Mathematical Sciences at the University of Southern California.

Throughout her career, Friedlander has focused on the mathematical analysis of partial differential equations (PDEs) arising in fluid dynamics. While the fundamental models are several centuries old, to date fluid dynamics remains the source of some of the most fascinating and challenging problems at the intersection of mathematics and physics. Without a doubt, the phenomenon of "turbulence" is chief among them. A unifying theme in Friedlander's research is an emphasis on problems of clear physical interest and importance.

Friedlander's impact on the field of mathematical fluid dynamics, and on the mathematical community as a whole, extends far beyond her research contributions. Prior to 1989, she opened bridges to the fluids communities behind the iron curtain. Since the early 1990s she has served in several leadership positions at the American Mathematical Society, including as Associate Secretary. For the past 15 years Friedlander has been the Editor in Chief of the Bulletin of the AMS, and more recently Friedlander was one of the key figures in the founding of the Mathematical Council of the Americas.

Susan is an exceptional mentor. Since the early stages of our careers, the authors of this paper were fortunate enough to collaborate with Susan, benefiting from her guidance, academic generosity, and perspective on mathematics as a whole. Susan has helped shape both our careers and our views of mathematics, and we are truly thankful for her inspiration, thoughtful guidance, and limitless positive energy.

In this review celebrating Friedlander's contributions we will focus on her work on hydrodynamic instability as it relates to the transition from laminar to turbulent flow, on dyadic models in fluid dynamics and Onsager's conjecture analyzing the transfer of energy in turbulent flows, and on magnetohydrodynamics as it relates to large-scale motions in Earth's fluid core.

\section{Equations of Fluid Dynamics}

The fundamental partial differential equations that describe the macroscopic properties of the motion of an incompressible, inviscid fluid with constant density are the
Euler equations:

$$
\begin{aligned}
\frac{\partial u}{\partial t}+(u \cdot \nabla) u+\nabla p & =0, \\
\nabla \cdot u & =0,
\end{aligned}
$$

with the initial condition

$$
u(x, 0)=u_{0}(x),
$$

for the unknown velocity vector field $u=u(x, t) \in \mathbb{R}^{d}$ and the pressure $p=p(x, t) \in \mathbb{R}$, where $x \in \mathbb{R}^{d}, t \in[0, \infty)$, and $d=2,3$. Despite the fact that Leonhard Euler introduced them in 1757, many basic questions concerning Euler equations in $d=3$ are still unresolved. For example, it is an outstanding problem to find out if solutions of the 3D Euler equations form singularities in finite time, from smooth initial data.

The equations modeling the macroscopic properties of viscous, incompressible, homogeneous fluids were formulated by Claude-Louis Navier (1822) and Sir George Stokes (1845). The Navier-Stokes equations that they derived are written as

$$
\begin{aligned}
\frac{\partial u}{\partial t}+(u \cdot \nabla) u+\nabla p & =v \Delta u, \\
\nabla \cdot u & =0,
\end{aligned}
$$

with the initial condition

$$
u(x, 0)=u_{0}(x),
$$

and appropriate boundary conditions. As with the Euler equations the theory of the Navier-Stokes equations in three dimensions is far from being complete. One of the major open problems is global existence and uniqueness of smooth solutions to the Navier-Stokes equations in 3D. This is one of the Millennium Problems of the Clay Mathematics Institute.

Beyond the Navier-Stokes and Euler equations many other models animate modern research in mathematical fluid dynamics. Of particular interest in Friedlander's work are the magnetohydrodynamics equations (cf. (34)-(37) below) and other equations arising in geophysics.

\section{Instabilities}

The late 1970s and early 1980 s were marked by the discovery of a new type of instability in incompressible fluidsthe so-called shortwave or broad-band instability. Such instabilities occur when a fluid rushes through a pipe leading to the formation of elliptical vortices near the walls. Although such vortices themselves are two dimensional, and in fact stable under two-dimensional perturbations, they are manifestly unstable when perturbed in the direction of their axes of rotation. Moreover, the frequency $\kappa$ of unstable modes corresponding to the same exponential rate $\lambda$,

$$
\mathcal{L} v \sim \lambda v, \quad v=e^{-i \kappa \cdot x} \phi(x),
$$


corresponds to a range of values $\kappa>\kappa_{0}$ instead of being uniquely determined by a dispersive relation $\kappa=$ $\kappa(\lambda)$. Hence, the term "broad-band." Grounded in numerous physical works by Orszag, Patera, Bayly, Pierrehumbert, Craik, Criminale, and others, these novel instabilities lacked a rigorous foundation presenting a unique challenge for the mathematical community in the early 1980 s.

2.1. The fast dynamo problem. A similar type of instability appears in the kinematic dynamo problem. This problem seeks to describe persistent growth of a magnetic field $H(x, t)$ transported by a given velocity field of electrically conducting fluid $u(x, t)$ in the limit of vanishing magnetic resistivity. Specifically, for $H$ satisfying the system

$$
\begin{aligned}
\frac{\partial H}{\partial t} & =-u \cdot \nabla H+H \cdot \nabla u+\varepsilon \Delta H, \\
\nabla \cdot H & =0,
\end{aligned}
$$

the dynamo is called fast if one has

$$
\limsup _{\varepsilon \rightarrow 0} \omega_{\varepsilon}>0,
$$

where $\omega_{\varepsilon}$ is the exponential type of the $C_{0}$-semigroup $\mathbf{G}_{t}^{\varepsilon}$ generated by (7)-(8). Similar to the fluid problem such instabilities are expected to be of highly oscillatory nature as the corresponding spectral problem $\mathcal{L}_{\varepsilon} H_{\varepsilon}=\lambda_{\varepsilon} H_{\varepsilon}$ would require an increasing range of frequencies as $\varepsilon \rightarrow 0$.

The groundbreaking works [FV91, FV92, VF93] marked the beginning of a productive collaboration of Friedlander with Misha Vishik, who developed a novel approach to the fast dynamo problem [Vis89]. This proved to be a universal tool to tackle a range of instability questions in fluids, geophysics, and magnetohydrodynamics. The approach is based on studying shortwave asymptotic expansions of the corresponding evolution semigroup or the associated Green's function. Here the general methodology is to reduce the evolution of an infinite-dimensional system to the leading order "core" dynamics. Remarkably in many cases the reduced dynamics is governed by a finite-dimensional system of ODEs.

In the context of the fast dynamo problem (7)-(9), the Green's function $G(x, y, t)$ of the evolution operator $\mathbf{G}_{t}^{\varepsilon}$ can be represented in Lagrangian coordinates

$$
\partial_{t} \varphi_{t}(x)=u\left(\varphi_{t}(x), t\right), \quad G\left(\varphi_{t}(x), y, t\right)=\Gamma(x, y, t)
$$

as the Fourier integral operator

$$
\Gamma(x, y, t)=\frac{1}{2 \pi \sqrt{\varepsilon}} \int_{\mathbb{R}^{n}} e^{\frac{i(x-y) \cdot \xi}{\sqrt{\varepsilon}}} b(x, \xi, t, \sqrt{\varepsilon}) \mathrm{d} \xi,
$$

where the symbol $b$ has an asymptotic expansion

$$
b(x, \xi, t, \sqrt{\varepsilon})=\sum_{n=0}^{\infty} b_{n}(x, \xi, t) \varepsilon^{n / 2} .
$$

Here the principal symbol $b_{0}$ which plays the determining role in exponential growth of the dynamics is obtained by

$$
\frac{\mathrm{d} b_{0}}{\mathrm{~d} t}=\partial u\left(\varphi_{t}, t\right) b_{0},
$$

the tangent push-forward transport map.

The technical analysis of the asymptotic series (10) is rather involved. Ultimately it connects the limiting exponential rate of $\mathbf{G}_{t}^{\varepsilon}$ as $\varepsilon \rightarrow 0$ over the energy space $L^{2}$ to that of the inviscid problem, and hence to the ODE (11). At the same time, the asymptotic behavior of (11) is well known. For steady states it is simply determined by the largest Lyapunov-Oseledets exponent of the underlying velocity field $u_{0}$ :

$$
\omega_{0}=\lim _{t \rightarrow \infty} \frac{1}{t} \log \sup _{x}\left|\partial \varphi_{t}(x)\right|
$$

(or otherwise the exponent of the corresponding cocycle family), which is positive if and only if the flow-map $\varphi_{t}(x)$ exhibits exponential stretching of its trajectories. The main result of [FV91] reads as follows.

Theorem 2.1. If the system (7)-(8) has a fast dynamo (9), then necessarily $\omega_{0}>0$, and in fact

$$
\limsup _{\varepsilon \rightarrow 0} \omega_{\varepsilon} \leq \omega_{0}
$$

Thus, a necessary condition for a fast dynamo is the presence of an instability in the underlying conducting fluid itself.

2.2. The geometric optics method. As we already described above the asymptotic methods developed by Friedlander and Vishik in attacking the fast dynamo problem proved to be applicable to a wide range of problems arising in fluid dynamics. Indeed, the works [VF93, FV92] laid the foundation to what is now called the geometric optics approach to shortwave instabilities, a particular case of which is the elliptic instability we mentioned in the beginning of this section.

To describe the method in more detail let us consider the example of the classical incompressible Euler system linearized around a given steady state $u_{0}$ :

$$
\begin{aligned}
\frac{\partial v}{\partial t} & =-u_{0} \cdot \nabla v-v \cdot \nabla u_{0}-\nabla p, \\
\nabla \cdot v & =0,
\end{aligned}
$$

where $v$ is the linear perturbation of $u_{0}$ and $p$ is the perturbed pressure, which plays the role of projecting the right side of (12) onto the space of divergent-free fields. We consider (12) with periodic boundary conditions, $x \in \mathbb{T}^{n}$, or the whole space $x \in \mathbb{R}^{n}, n \geq 2$. The method seeks to find effective dynamics of a localized oscillatory wave written in the form of a geometric optics ansatz

$$
v(x, t)=b(x, t) e^{i S(x, t) / \varepsilon}+O(\varepsilon) .
$$


If initially $b(0)=b_{0}$ is localized near position $x_{0}$, and the frequency of initial oscillation is $\xi$, i.e., $S(0)=S_{0}=\xi \cdot x$, we obtain a new wave at time $t$ approximately localized near the Lagrangian particle $x(t)=\varphi_{t}\left(x_{0}\right)$. Plugging this ansatz into the Euler system (12) one reads off the leading order evolution of the amplitude $b$ and phase $S$ in the Lagrangian coordinates of the underlying field $u_{0}$ :

$$
\frac{\mathrm{d} S}{\mathrm{~d} t}=0, \quad \frac{\mathrm{d} b}{\mathrm{~d} t}=-\partial u_{0}(x) b+2\left\langle\xi, \partial u_{0}(x) b\right\rangle \frac{\xi}{|\xi|^{2}},
$$

where $\xi=\nabla S$ is the frequency covector. To write the system in closed form we replace the transport of $S$ by the transport of the frequency vector, and the resulting system reads

$$
\left\{\begin{array}{l}
\frac{\mathrm{d} x}{\mathrm{~d} t}=u_{0}(x), \\
\frac{\mathrm{d} \xi}{\mathrm{d} t}=-\partial^{\top} u_{0}(x) \xi, \\
\frac{\mathrm{d} b}{\mathrm{~d} t}=-\partial u_{0}(x) b+2\left\langle\xi, \partial u_{0}(x) b\right\rangle \frac{\xi}{|\xi|^{2}},
\end{array}\right.
$$

supplemented by initial conditions $x(0)=x_{0}, \xi(0)=\xi_{0}$, $b(0)=b_{0}$, and the incompressibility constraint $b_{0} \cdot \xi_{0}=0$. This is a so-called bicharacteristic-amplitude system (BAS for short). From a dynamical viewpoint the first two equations represent a bicharacteristic flow on the tangent bundle of the fluid domain $\Omega=T^{*} \mathbb{T}^{n}$, denoted $\chi_{t}(x, \xi)$, and the last amplitude equation represents evolution of a vector $b$ on the fiber bundle over $\Omega$ with fibers given by orthogonal planes $\pi^{-1}(x, \xi)=\{b: b \cdot \xi=0\}$. Thus, $b\left(x_{0}, \xi_{0}, b_{0}, t\right)=\mathbf{B}_{t}(x, \xi) b_{0}$ is a cocycle family of maps over the flow $\chi$.

The asymptotic expansion of the Euler semigroup $\mathbf{G}_{t}$, defined by (12)-(13), is dominated by the $B$-cocycle playing the role of the principal symbol

$$
\mathbf{G}_{t}=\mathbf{P} \boldsymbol{\Phi}_{t} \mathrm{Op}\left[\mathbf{B}_{t}\right]+\mathbf{K}_{t},
$$

where $\mathbf{P}$ is the Leray projection onto the divergence-free fields, $\boldsymbol{\Phi}_{t} v=v \circ \varphi_{-t}$,

$$
\mathrm{Op}\left[\mathbf{B}_{t}\right] v(x)=\int e^{i(x-y) \cdot \xi} \mathbf{B}_{t}(y, \xi) v(y) \mathrm{d} y \mathrm{~d} \xi
$$

is the leading order pseudodifferential operator, and $\mathbf{K}_{t}$ is a similar operator of order -1 . For the high frequency waves the frequency localization of pseudodifferential operator Op $\left[\mathbf{B}_{t}\right]$ leads precisely to the ansatz (14) which becomes justified a posteriori.

The shortwave instabilities can now be studied by looking into the Lyapunov spectrum of the BAS whose maximal exponent is given by

$$
\mu=\lim _{t \rightarrow \infty} \frac{1}{t} \log \sup _{x_{0}, \xi_{0}, b_{0} \cdot \xi_{0}=0,\left|b_{0}\right|=1}\left|B_{t}\left(x_{0}, \xi_{0}\right) b_{0}\right| .
$$

The main result of [VF93] establishes a direct relationship between the growth rate of the BAS and the growth rate of the Euler dynamics in the energy space.

Theorem 2.2. Let $\omega_{0}$ denote the exponential rate of the semigroup $\mathbf{G}_{t}$ in $L^{2}$. Then

$$
\omega_{0} \geq \mu
$$

The high frequency asymptotic relationship between $\mathbf{G}_{t}$ and $\mathbf{B}_{t}$ makes it possible to relate shortwave instabilities to the essential spectrum of the semigroup. The BAS was found to be fully descriptive of the essential spectrum in later works. For particular flows, however, Theorem 2.2 proved to be extremely versatile in many different situations. For example, for the aforementioned elliptic vortices, locally given by $u_{0}=\left(a^{2} y,-b^{2} x, 0\right)$, the growth of the BAS becomes a Floquet problem over time-periodic elliptic trajectories. This case was also studied in works of Lifschitz and Hameiri around the same time. The amplitudes $b$ become unstable in directions pointing off of the $x y$-plane, which is consistent with the empirical observations of Orszag, Patera, and others. A systematic study of the BAS and various dynamic scenarios leading to instabilities was performed in [FV92]. First, in 2D, the quantity $|b||\xi|$ is conserved. Hence, $\mu$ is related precisely to the exponential stretching of the underlying field $u_{0}$. Here the cotangent cocycle associated with the $\xi$-equation has the same Lyapunov spectrum as that of the tangent cocycle (11). Thus, $\mu=\omega_{0}$ in this case. In particular, all parallel shear flows are shortwave stable. In $3 \mathrm{D}$, the analogue of this law is conservation of the volume

$$
\operatorname{Vol}\left(b^{\prime}, b^{\prime \prime}, \xi\right)=\left(b^{\prime} \times b^{\prime \prime}\right) \cdot \xi
$$

for any pair of amplitudes $b^{\prime}, b^{\prime \prime}$ over the same frequency $\xi$. Hence, in 3D we obtain

$$
\mu \geq \frac{1}{2} \omega_{0} .
$$

In any case, exponential stretching makes the flow spectrally unstable. On the other hand, some integrable flows $u_{0}$, where $u_{0} \times\left(\nabla \times u_{0}\right)=\nabla B$, on nondegenerate level tori of the Bernoulli function $B$ are found to be stable, namely $\mu=0$. Geometric instability criteria for vortex rings without swirl were provided as well.

In several subsequent works (see [FSV97, FS05] and references therein), Friedlander expanded the geometric optics method to a range of models appearing in geophysics and magnetohydrodynamics. In all these cases the underlying bicharacteristic flow remains the same but the amplitude equation changes according to a simple recipe-it captures the principal symbol of the linearization:

$$
\frac{\mathrm{d} b}{\mathrm{~d} t}=a_{0}(x, \xi) b, \quad \mathcal{L}=-u_{0} \cdot \nabla+\mathrm{Op}\left[a_{0}\right]+\mathrm{Op}\left[a_{1}\right]+\cdots .
$$


Thus, for the surface quasigeostrophic equation describing evolution of a potential temperature on a horizontal surface the $b$-equation reads

$$
\frac{\mathrm{d} b}{\mathrm{~d} t}=i \frac{\xi^{\perp} \cdot \nabla \theta_{0}(x)}{|\xi|} b
$$

where $\theta_{0}$ is the underlying steady temperature. In this case the essential spectrum is neutral, $\mu=0$. For density stratified fluids we have

$$
\begin{aligned}
\frac{\mathrm{d} b}{\mathrm{~d} t} & =\left(2 \frac{\xi \otimes \xi}{|\xi|^{2}}-\mathrm{id}\right) \partial u(x) b+r\left(\mathrm{id}-\frac{\xi \otimes \xi}{|\xi|^{2}}\right) \nabla \Phi(x), \\
\frac{\mathrm{d} r}{\mathrm{~d} t} & =-b \cdot \nabla \rho_{0}(x) .
\end{aligned}
$$

Here $\Phi$ is the gravitational potential. The kinematic dynamo falls under the same scheme and yields (11). Camassa-Holm (Euler- $\alpha$ ) gives

$$
\frac{\mathrm{d} b}{\mathrm{~d} t}=\left(\frac{\xi \otimes \xi}{|\xi|^{2}}-\mathrm{id}\right) \partial u^{\top}(x) b+\frac{\xi \otimes \xi}{|\xi|^{2}} \partial u(x) b,
$$

and for inviscid systems of nonrelativistic superconductivity we have

$$
\frac{\mathrm{d} b}{\mathrm{~d} t}=\left(2 \frac{\xi \otimes \xi}{|\xi|^{2}}-\mathrm{id}\right) \partial u(x) b+\left(\mathrm{id}-\frac{\xi \otimes \xi}{|\xi|^{2}}\right) B \times b .
$$

Numerous other applications of the theory were found to non-Newtonian fluids also. The reach of the method proved to be truly astonishing.

2.3. From linear to nonlinear instability. Justification of the linearization procedure for inviscid fluids remains a very challenging problem to this day. Providing an explicit bound on the "bad" essential part of the spectrum given by exponent $\mu$ is a helpful tool to prove a range to sufficient conditions for the analogue of the Lyapunov theorygoing from linear to nonlinear instability [FSV97]. In several subsequent works Friedlander established several pioneering results in this direction. First, in 2D if there is a point spectrum (exact eigenvalue) beyond $\mu$, i.e.,

$$
\mathcal{L} v=\lambda v, \quad \operatorname{Re} \lambda>\mu,
$$

then the underlying steady flow $u_{0}$ is unstable in the energy norm [VF03]. Construction of flows with oscillatory laminar regions that fulfill this condition have been provided in Friedlander's works with Yudovich. In the region outside of the essential spectrum, in fact one can also construct unstable invariant manifolds by analogy with finitedimensional theory and dissipative systems as was done later in works of Lin and Zeng. Next, for the linearized Navier-Stokes system

$$
\begin{aligned}
\frac{\partial v}{\partial t} & =-u_{0} \cdot \nabla v-v \cdot \nabla u_{0}-\nabla p+\varepsilon \Delta v, \\
\nabla \cdot v & =0
\end{aligned}
$$

both in $2 \mathrm{D}$ and in $3 \mathrm{D}$, those dominant eigenvalues reappear for small viscosities $\varepsilon \rightarrow 0$ in a strong spectral limit: for any eigenvalue of the linearized Euler system with $\operatorname{Re} \lambda>\mu$ and $\varepsilon$ sufficiently small the Navier-Stokes system gains point spectrum in a vicinity of $\lambda$ with the same multiplicity, and moreover the Riesz projection $\mathbf{P}^{\varepsilon}$ corresponding to those spectral subspaces near $\lambda$ tends to that of the Euler equation $\mathbf{P}^{0}$ in the uniform operator topology. This result proves to be particularly interesting in view of the fact that the nonlinear Navier-Stokes system inherits instability in $L^{3}$ (and in fact any $L^{p}$ for $p>1$ ) from the linearization, a classical result of Yudovich. Thus, any steady flow in $3 \mathrm{D}$ that has inviscid spectrum beyond $\mu$ becomes nonlinearly unstable in the vanishing viscosity sense.

\section{Dyadic Models}

One way to gain an understanding of certain aspects of the equations of fluid motion is to introduce toy models which share properties with the actual equations, but are simpler to analyze. During the last two decades, the work of Friedlander has shaped studies of so-called dyadic models of the fluid equations, which simulate the energy cascade through dyadic frequency shells. ${ }^{1}$ In these models, the nonlinearity of the Euler equations $(u \cdot \nabla) u$ is simplified so that only local interactions between neighboring scales are considered. However, simplifications of the nonlinear term vary, and as a consequence the models differ. Some of the first examples of models of this type were derived by Desnyanskiy and Novikov in the context of oceanography, and by Gledzer, whose model was subsequently generalized by Ohkitani and Yamada (and is now known as the GOY model).

The dyadic models that Friedlander explored are designed to share with the actual equations of fluid motion the scaling of the nonlinear term in $3 \mathrm{D}$ (which we motivate in the next subsection) and the following properties:

- A skew-symmetry property of the nonlinear term,

$$
\langle(u \cdot \nabla) u, u\rangle_{L^{2}\left(\mathbb{R}^{3}\right)}=0 .
$$

- Conservation of energy for the classical solutions to the Euler equations,

$$
\|u(\cdot, T)\|_{L^{2}}^{2}=\left\|u_{0}\right\|_{L^{2}}^{2},
$$

which is a consequence of (18) and the divergence-free condition, as can be seen by pairing the Euler equation (1) with $u$ in the $L^{2}$ sense.

- Decay of energy for classical solutions to the Navier-Stokes equations (4)-(6),

$$
\|u(\cdot, T)\|_{L^{2}}^{2}=\left\|u_{0}\right\|_{L^{2}}^{2}-2 \nu \int_{0}^{T}\langle-\Delta u, u\rangle .
$$

${ }^{1}$ Specifically, a jth dyadic shell refers to a region where the Fourier frequency $\xi$ lies in the annular domain $2^{j-1} \leq|\xi| \leq 2^{j}$. The fluid velocity in the jth dyadic shell is modeled with a single representative, $u_{j}$. 
Broadly speaking, dyadic models provide a framework for studying specific aspects of turbulence theory, while being mathematically accessible. Moreover, in some instances these models motivated results on actual equations of fluid motion, as was the case in e.g. [CCFS08].

3.1. Introducing a dyadic model. Let us now recall a version of a dyadic model from [CFP07]. This model was inspired by a wavelets model introduced in [KP02] as a tool to help guide a partial regularity result for actual NavierStokes equations with hyperdissipation. We start by briefly revisiting the wavelets model.

First, we recall some terminology from [KP02]. A cube $Q$ in $\mathbb{R}^{3}$ is called a dyadic cube if its sidelength is an integer power of $2,2^{l}$, and the corners of the cube are on the lattice $2^{l} \mathbb{Z}^{3}$. Let $D$ denote the set of dyadic cubes in $\mathbb{R}^{3}$. Let $\mathcal{D}_{j}$ denote the subset of dyadic cubes having sidelength $2^{-j}$. Then the parent of $Q$, denoted by $P Q$, is introduced as the unique dyadic cube in $\mathcal{D}_{j(Q)-1}$ which contains $Q$. On the other hand, one defines $\mathcal{C}^{k}(Q)$, the $k$ th-order grandchildren of $Q$, to be the set of those cubes in $\mathcal{D}_{j(Q)+k}$ which are contained in $Q$.

The modeling starts by replacing a vector-valued function $u$ by a scalar-valued one. An orthonormal family of wavelets is denoted by $\left\{w_{Q}\right\}$, with $w_{Q}$ the wavelet associated to the spatial dyadic cube $Q \in \mathcal{D}_{j}$. Then $u$ can be represented as

$$
u(x, t)=\sum_{Q} u_{Q}(t) w_{Q}(x)
$$

Note that due to spatial localization of $w_{Q}$,

$$
\left\|w_{Q}\right\|_{L^{\infty}} \sim 2^{\frac{3 j(Q)}{2}} .
$$

On the other hand

$$
\left\|\nabla w_{Q}\right\|_{L^{2}} \sim 2^{j}
$$

Having in mind (21) and (22), a cascade-down operator is defined through its $Q$ th coefficient as follows:

$$
\left(C_{d}(u, v)\right)_{Q}=2^{\frac{5 j(Q)}{2}} u_{P Q} v_{P Q},
$$

with the scaling $2^{5 j(Q)}$ that reflects the upper bound on the nonlinear term implied by (21)-(22). Similarly, a cascadeup operator is defined as the adjoint of $C_{d}(u, v)$ via

$$
\left(C_{u}(u, v)\right)_{Q}=2^{\frac{5(j(Q)+1)}{2}} u_{Q} \sum_{Q^{\prime} \in C^{1}(Q)} v_{Q^{\prime}}
$$

Then the cascade operator is introduced as

$$
C(u, v)=C_{u}(u, v)-C_{d}(u, v) .
$$

Having defined the Laplacian as $-\Delta\left(w_{Q}\right)=2^{2 j} w_{Q}$, one introduces the following model equations:

- Dyadic Euler equation:

$$
\frac{\mathrm{d} u}{\mathrm{~d} t}+C(u, u)=0
$$

- Dyadic Navier-Stokes equation:

$$
\frac{\mathrm{d} u}{\mathrm{~d} t}+C(u, u)-\Delta u=0 .
$$

By construction of the cascade operators, we have $\left\langle C_{u}(u, u), u\right\rangle=\left\langle C_{d}(u, u), u\right\rangle$, which implies the skewsymmetry property of the operator $C$,

$$
\langle C(u, u), u\rangle=0 .
$$

A simple consequence of (23) is conservation of energy for the dyadic Euler equations and decay of energy for the dyadic Navier-Stokes equations, at least at the formal level (for sufficiently regular solutions).

The above dyadic models are special cases of the following infinite system of coupled ordinary differential equations, which was studied by Friedlander:

$$
\frac{\mathrm{d}}{\mathrm{d} t} a_{j}+v 2^{2 j} a_{j}-2^{c(j-1)} a_{j-1}^{2}+2^{c j} a_{j} a_{j+1}=f_{j}
$$

for $j=0,1,2, \ldots$, where $a_{-1}=0, c$ is a positive parameter related to intermittency, and $\frac{1}{2} a_{j}^{2}$ represents the total energy in the frequencies of order $2^{j}$. The force $f$ is such that $f_{0}>0$ and $f_{j}=0$ for all $j>0$, so that the energy is pumped on low modes.

As we have seen above, the model preserves many features of the fluid equations, while the nonlinearity is simplified by considering only local interactions between scales. Moreover, the choice of the constant $c=5 / 2$ ensures that the nonlinearity in the dyadic model obeys the same $L^{2}$-based estimates as Euler (see (19)) and NavierStokes equations (see (20)). Thanks to these $L^{2}$-based estimates and a certain monotonicity present in the model, a finite time blow-up was exhibited for the inviscid dyadic model [KP05], as well as the viscous dyadic model with some "small" degrees of dissipation [KP05] or large values of $c$ [Che08]. For instance, solutions blow up when $c>3$, in which case the dyadic model scales as 4+-dimensional Navier-Stokes equations. Such a monotonicity property resembles monotonicity of certain quantities present in socalled "cooperative" systems (see for example the work of Palais and the work of Bernoff and Bertozzi where singularities in a modified Kuramoto-Sivashinsky equation were identified). Finite time blow-up in the inviscid case was sharpened by Kiselev and Zlatoš. A three-dimensional vector model for the incompressible Euler equations was introduced in [FP04], which is similar in some features to a discretized approximate model constructed by Dinaburg and Sinai for the Navier-Stokes equations in Fourier space. It was shown in [FP04] that for special initial data the evolution equations of the divergence-free vector model reduced to the scalar dyadic Euler system and finite time blow-up occurs in this model for the three-dimensional incompressible Euler equations. This was a brief snapshot of 
results for dyadic models around 2005, when Susan Friedlander initiated the study of phenomena related to turbulence at the level of dyadic models.

3.2. Onsager's and Kolmogorov's conjectures. Up to now we discussed conservation of energy at a formal level, i.e., for sufficiently regular solutions to Euler equations (1). However one might wonder about the minimal level of regularity of a solution to the Euler equation that guarantees conservation of energy. In fact this seemingly naive question is connected with the statistical theories of turbulence developed by Kolmogorov (1941) and Onsager (1949). In their seminal works, it is suggested that an appropriate mathematical description of three-dimensional turbulent flow is given by weak solutions of the Euler equations which are not regular enough to conserve energy. Onsager conjectured that for the velocity Hölder exponent $h>1 / 3$ the energy is conserved ${ }^{2}$ and that this ceases to be true for $h \leq 1 / 3$. This latter phenomenon is now called turbulent or anomalous dissipation. Kolmogorov's theory predicts that in a fully developed turbulent flow the energy spectrum $E(|k|)$ in the inertial range is given by

$$
E(|k|)=c_{0} \bar{\epsilon}^{2 / 3}|k|^{-5 / 3}
$$

where $\bar{\epsilon}$ is the average of the energy dissipation rate.

While the rigidity part of Onsager's conjecture (namely the regime corresponding to the conservation of energy) has been well understood due to works of Eyink and Constantin-E-Titi-prior to works on dyadic models-the flexibility part represented a challenge for a long period of time. Thanks to advances in the method of convex integration due to De Lellis-Székelyhidi, the flexibility part of the Onsager conjecture for the Euler equation has been very recently settled by Isett, and by Buckmaster-De LellisSzékelyhidi-Vicol for dissipative solutions. However, the state of the puzzle regarding the flexible part of the conjecture was completely open back in 2007. In that context, the dyadic model (24) provided a mathematical laboratory for addressing the phenomena predicted by Onsager and Kolmogorov.

More precisely, in [CFP07, CFP10] Friedlander et al. showed that the inviscid $(\nu=0)$ dyadic model possesses a unique fixed point $\tilde{a}$, whose energy spectrum $\mathcal{S}(\kappa) \sim$ $\varepsilon^{2 / 3} \kappa^{-8 / 3}$, which is just on the borderline of the Sobolev space $H^{5 / 6}$, where the $H^{s}$ Sobolev space is equipped with the norm

$$
\|a\|_{H^{s}}^{2}=\sum_{j=0}^{\infty} 2^{2 s j} a_{j}^{2} .
$$

This showed that all the solutions of the dyadic Euler model stop satisfying energy equality at some time (which

\footnotetext{
${ }^{2} A$ very rough motivation for the appearance of the Hölder exponent $1 / 3$ is "sharing" of one derivative among three copies of velocity $u$ in the skewsymmetry relation (18), which has a crucial role in producing energy equalities (19) and (20).
}

resolved Onsager's conjecture in the negative direction), and the long-time behavior is exactly as predicted by Kolmogorov's theory of turbulence, but with extreme (or what we now call fully intermittent) energy spectrum. In fact, as it was observed in [CF09], the dyadic model (24) covers the whole intermittency range $d \in[0,3]$, where the intermittency dimension $d$ is connected to the parameter $c$ as $c=1+\frac{3-d}{2}$ (see Subsection 3.3). Then

Theorem 3.1 ([CFP07, CFP10]). The following hold for the dyadic system (24) in the inviscid case $v=0$ :

1. Every regular solution (defined to be a solution with bounded $H^{5 / 6}$ norm) satisfies the energy equality.

2. There exists a unique fixed point a to (24), which is a global attractor. The fixed point is not in $H^{5 / 6}$. In fact, it lies exactly in the space $B_{3, \infty}^{1 / 3}$ (defined in (31) below), which takes into account intermittency.

3. The energy spectrum of the fixed point

$$
\mathcal{S}(\kappa) \sim \varepsilon^{2 / 3} \kappa^{-\frac{8-d}{3}},
$$

where $\tilde{a}_{0} f_{0}$ is the energy input rate, that corresponds to the anomalous energy dissipation rate.

4. Every solution blows up in finite time in $H^{5 / 6}$ and in $B_{3, \infty}^{1 / 3+\epsilon}$ for any $\epsilon>0$.

5. The $H^{S}$ norms of every solution are locally square integrable in time for $s<5 / 6$, and every solution eventually dissipates energy.

We note that the relevance of the Sobolev exponent $5 / 6$ stems from three copies of modeled velocity "sharing" the scaling of the localized coefficient $2^{5 / 2}$ in the skewsymmetry property for the dyadic nonlinear term (23). The existence of a global attractor for an inviscid system, at first, seems surprising. However it is exactly consistent with the concept of anomalous or turbulent dissipation conjectured by Onsager.

The relation between the fixed points of inviscid and viscous dyadic models is as follows.

Theorem 3.2 ([CF09]). The following hold for the dyadic system (24) in the viscous case $v>0$ :

1. The global attractor for the viscous dyadic model is a fixed point $\tilde{a}^{\nu}$.

2. The fixed point of the viscous system $\tilde{a}^{\nu}$ converges in $l^{2}$ to the fixed point of the inviscid system a as $\nu \rightarrow 0$. Moreover, the energy dissipation rate converges to the anomalous energy dissipation rate of the inviscid system, i.e.,

$$
\lim _{\nu \rightarrow 0} \varepsilon^{\nu}=\langle f, \tilde{a}\rangle=\varepsilon,
$$

where

$$
\varepsilon^{\nu}=\nu\left\|\tilde{a}^{\nu}\right\|_{H^{1}}^{2}=\left\langle f, \tilde{a}^{\nu}\right\rangle
$$

is the energy dissipation rate. 
3.3. From the dyadic model to the full equations. One of the main features of the dyadic Navier-Stokes model, the forward energy cascade, leads to the question of whether solutions satisfy the energy equality. The nonlinear term in the dyadic model is skew-symmetric by construction, and hence one immediately obtains

$$
\frac{1}{2} \frac{\mathrm{d}}{\mathrm{d} t} \sum_{i=0}^{j} a_{i}^{2}=-\Pi_{j}-\nu \sum_{i=0}^{j} 2^{2 i} a_{i}^{2}+\sum_{i=0}^{j} f_{i} a_{i},
$$

where the flux is defined as

$$
\Pi_{j}=2^{\frac{5}{2} j} a_{j}^{2} a_{j+1},
$$

where we again chose $c=5 / 6$. Passing to the limit as $j \rightarrow$ $\infty$, it follows that every solution $a \in L^{3}\left(0, T ; H^{5 / 6}\right)$ satisfies the energy equality

$$
\begin{aligned}
\sum_{i=0}^{\infty} a_{i}^{2}(t)= & \sum_{i=0}^{\infty} a_{i}^{2}\left(t_{0}\right) \\
& +\int_{t_{0}}^{t}\left[-v \sum_{i=0}^{\infty} 2^{2 i} a_{i}^{2}+\sum_{i=0}^{\infty} f_{i} a_{i}\right] \mathrm{d} s
\end{aligned}
$$

for all $0 \leq t_{0} \leq t \leq T$. Surprisingly, this result was not known for the fluid equations at that time, so Friedlander and collaborators extended it to the Navier-Stokes equations in [CSF12].

As we have seen in Section 3.2, a simplified dyadic model (24) mimicked highly nontrivial predictions for real equations, raising the following questions. First, can we rigorously justify the derivation of the model? Second, what is the meaning of the parameter $c$ that affects the energy spectrum, which ranges from classical Kolmogorov's $5 / 3$ to the extreme $8 / 3$ power law? This started a fruitful series of works on obtaining optimal bounds for the energy flux and incorporating a notion of intermittency in the mathematical studies of fluid equations.

Consider the Navier-Stokes equations (4) for the motion of a three-dimensional incompressible viscous fluid. Define

$$
u_{\leq \lambda_{j}}=u * \mathcal{F}^{-1}\left(\psi\left(\cdot 2^{-j}\right)\right),
$$

where $\psi(\xi)$ is a smooth nonnegative function supported in the ball of radius one centered at the origin and such that $\psi(\xi)=1$ for $\xi \leq 1 / 2$, and $\mathcal{F}$ is the Fourier transform. The energy flux due to nonlinear interactions through the sphere of radius $\lambda_{j}=2^{j}$ is defined as (see [CCFS08])

$$
\Pi_{j}=-\int_{\mathbb{R}^{3}}(u \cdot \nabla) u \cdot\left(u_{\leq \lambda_{j}}\right)_{\leq \lambda_{j}} \mathrm{~d} x .
$$

Using the test function $\left(u_{\leq \lambda_{j}}\right)_{\leq \lambda_{j}}$ in the weak formulation of the Navier-Stokes equations we obtain

$$
\frac{1}{2} \frac{\mathrm{d}}{\mathrm{d} t}\left\|u_{\leq \lambda_{j}}\right\|_{2}^{2}=-\Pi_{j}-v\left\|\nabla u_{\leq \lambda_{j}}\right\|_{2}^{2}+\left\langle f_{\leq \lambda_{j}}, u_{\leq \lambda_{j}}\right\rangle .
$$

In [CCFS08], Cheskidov, Constantin, Friedlander, and Shvydkoy obtained the following new bounds on the nonlinear term in (4):

$$
\left|\Pi_{j}\right| \lesssim \sum_{i=-1}^{\infty} \lambda_{|j-i|}^{-\frac{2}{3}} \lambda_{i}\left\|u_{i}\right\|_{3}^{3},
$$

where $u_{j}=u_{\leq j+1}-u_{\leq j}$ is the Littlewood-Paley projection of $u$. This estimate employing the Littlewood-Paley decomposition produced not only a sharpening of the conditions under which there is no anomalous dissipation, but also provides detailed information concerning the cascade of energy through frequency space. More precisely, it shows that the energy flux $\Pi$ through the sphere of radius $\kappa$ is controlled primarily by scales of order $\kappa$. The estimate also showed that a critical space for solutions in which the energy equality is guaranteed, Onsager's space, is $B_{3, \infty}^{1 / 3}$ for the Navier-Stokes equations, and $B_{3, c_{0}}^{1 / 3}$ for the Euler equations.

Now define $a_{j}=\left\|u_{j}\right\|_{2}$, so that $\frac{1}{2} a_{j}^{2}$ represents the energy in the dyadic shell of radius $2^{j}$. In order to mimic the flux estimate (29), we need to pass from $L^{3}$ to $L^{2}$, which can be done thanks to Bernstein's inequality:

$$
a_{j}^{3} \lesssim\left\|u_{j}\right\|_{3}^{3} \lesssim \lambda_{j}^{\frac{3}{2}} a_{j}^{3} .
$$

To capture the whole range of possible saturations of the Bernstein inequality, define an intermittency parameter $d \in[0,3]$, which, roughly speaking, represents the dimension of the set occupied by eddies, such that

$$
\left\|u_{j}\right\|_{3} \sim \lambda_{j}^{\frac{3-d}{6}} a_{j} .
$$

Then it is natural to define the Besov norm of $a$ as

$$
\|a\|_{B_{3, \infty}^{s}}=\sup _{j} 2^{j\left(s+\frac{3-d}{6}\right)} a_{j} .
$$

This combined with the locality of (29) motivates the following model for the energy flux:

$$
\Pi_{j}=\lambda_{j}^{1+\frac{3-d}{2}} a_{j}^{2} a_{j+1} .
$$

Here $d=3$ corresponds to the so-called Kolmogorov's regime where eddies occupy the whole space (or lower bound on $\left\|u_{j}\right\|_{3}$ in (30)) and $d=0$ is the case of extreme intermittency (or upper bound in (30)). Motivated by (28), subtracting subsequent equations (26) (for $j$ and $j-1$ ) gives

$$
\frac{1}{2} \frac{\mathrm{d}}{\mathrm{d} t} a_{j}^{2}=\Pi_{j-1}-\Pi_{j}-v \lambda_{j}^{2} a_{j}^{2}+f_{j} a_{j},
$$

which is exactly the dyadic model (24) with $c=1+\frac{3-d}{2}$ by definition of the flux. 


\section{The Magnetogeodynamo}

The geodynamo is the process by which the rotating, convecting, electrically conducting molten iron in Earth's fluid core maintains the geomagnetic field against ohmic decay. The convective processes in the core that produce the velocity fields required for this dynamo action are a combination of thermal and compositional convection. A detailed description of the dynamo problem requires the examination of the three-dimensional partial differential equations governing incompressible magnetohydrodynamics (MHD) under the effect of Coriolis, Lorentz, and gravity forces (see the system (34)-(37) below). The system also possesses thermal source terms which model radioactive decay within Earth's core, and can have an essentially stochastic character. The mathematical statement of the geodynamo problem asks whether there are initial data for the MHD system for which the evolution of the magnetic field grows for a sufficiently long time, i.e., the existence of instabilities. These instabilities are also expected to play a fundamental role in magnetostrophic turbulence and turbulent dynamo theory [ML94].

Due to its complexity, in order to simulate this system, current computational limitations require parameter choices that are several orders of magnitude larger than what is physically realistic. It is therefore reasonable to attempt to gain some insight into the geodynamo by considering a reduction of the full MHD equations to a system that is more tractable, but still maintains some of the key physical features. The magnetogeostrophic (MG) equation proposed by Moffatt and Loper [ML94] (see (37) and (41) below) is one such model, which has gained significant interest in the mathematical community, mostly due to Friedlander's work on this subject. During the past decade Friedlander and her collaborators have gone from laying down the mathematical foundations of the MG equations, to proving delicate results about the long-time dynamics of solutions, the instability of its steady states as it relates to the geodynamo, and to rigorously deriving the model from the small parameter regime postulated in its physical derivation.

4.1. Derivation of the MG model. For simplicity of notation, assume that the axis of rotation and the gravity are aligned in the direction of the Cartesian vector $e_{3}$. Moffatt and Loper [ML94] furthermore assume that the magnetic field is the sum of an underlying purely toroidal constant field $B_{0} e_{2}$ and a perturbation field $b(x, t)$. The fluid velocity vector field is denoted by $u(x, t)$, while the buoyancy scalar field as $\theta(x, t)$. In the rotating frame of reference the MHD system becomes:

$$
\begin{aligned}
& N^{2}\left[R_{o}\left(\partial_{t} u+u \cdot \nabla u\right)+e_{3} \times u\right]+\nabla P \\
& =e_{2} \cdot \nabla b+R_{m} b \cdot \nabla b+N^{2} \theta e_{3}+v \Delta u,
\end{aligned}
$$

$$
\begin{aligned}
& R_{m}\left[\partial_{t} b+u \cdot \nabla b-b \cdot \nabla u\right]=e_{2} \cdot \nabla u+\Delta b, \\
& \nabla \cdot u=0, \quad \nabla \cdot b=0, \\
& \partial_{t} \theta+u \cdot \nabla \theta=\kappa \Delta \theta+S .
\end{aligned}
$$

Here $S(x, t)$ is a given thermal source due to radioactive decay acting on the system, and naturally may be considered to include stochastic components. The dimensionless physical parameters that appear above are: the inverse Elsässer number $N^{2}$, the Rossby number $R_{o}$, the magnetic Reynolds number $R_{m}$, the inverse Peclet number $\kappa$, and the inverse square of the Hartman number $\nu$.

The physical postulate of the Moffatt-Loper MG model is that slow cooling of the Earth leads to slow solidification of the liquid metal core onto the solid inner core, releasing latent heat of solidification which drives compositional convection in the fluid core. Based on this physical postulate, arguments are given in [ML94] for the appropriate ranges of the aforementioned parameters: $N \approx 1$ and $R_{o}, R_{m} \ll 1$. The MG model is obtained by setting $N=1$ and passing $R_{o}, R_{m} \rightarrow 0$ in (34)-(36), equations which simplify to ${ }^{3}$

$$
\begin{aligned}
& e_{3} \times u=-\nabla P+e_{2} \cdot \nabla b+\theta e_{3}+\nu \Delta u, \\
& 0=e_{2} \cdot \nabla u+\Delta b, \\
& \nabla \cdot u=0, \quad \nabla \cdot b=0 .
\end{aligned}
$$

The linear system of equations (38)-(40) determine the vector fields $u$ and $b$ in terms of the scalar buoyancy $\theta$, encoding the vestiges of the physics in the problem: Coriolis force, Lorentz force, and gravity. Further vector manipulations of (38)-(40) give the expression

$$
\begin{aligned}
& {\left[\left(e_{3} \cdot \nabla\right)^{2} \Delta+\left[\nu \Delta^{2}-\left(e_{2} \cdot \nabla\right)^{2}\right]^{2}\right] u} \\
& \quad=\left(e_{3} \cdot \nabla\right) \Delta\left(e_{3} \times \nabla \theta\right) \\
& \quad-\left[v \Delta^{2}-\left(e_{2} \cdot \nabla\right)^{2}\right] \nabla \times\left(e_{3} \times \nabla \theta\right)
\end{aligned}
$$

which allows us to compute $u$ as a function of $\theta$, under the model's self-consistency assumption that both $\theta$ and $u$ have zero vertical mean. ${ }^{4}$ Note that all the differential operators appearing in (41) have constant coefficients. Thus, it is convenient to rewrite (41) as $u=M_{\nu}[\theta]$, where $M_{\nu}$ is a vector Fourier multiplier operator with associated symbol $\hat{M}_{\nu}$ given by

$$
\begin{aligned}
\hat{M}_{\nu 1}(k) & =\left(k_{2} k_{3}|k|^{2}-k_{1} k_{3}\left(k_{2}^{2}+\nu|k|^{4}\right)\right) D_{\nu}(k)^{-1}, \\
\hat{M}_{\nu 2}(k) & =\left(-k_{1} k_{3}|k|^{2}-k_{2} k_{3}\left(k_{2}^{2}+\nu|k|^{4}\right)\right) D_{\nu}(k)^{-1}, \\
\hat{M}_{\nu 3}(k) & =\left(\left(k_{1}^{2}+k_{2}^{2}\right)\left(k_{2}^{2}+\nu|k|^{4}\right)\right) D_{\nu}(k)^{-1}, \\
D_{\nu}(k) & =|k|^{2} k_{3}^{2}+\left(k_{2}^{2}+\nu|k|^{4}\right)^{2}
\end{aligned}
$$

\footnotetext{
${ }^{3}$ The orders of $v$ and $x$ are speculative, but likely very small. For the moment we keep them as free parameters, which allows us to pass $\nu, \kappa \rightarrow 0$ later on in the analysis.

${ }^{4}$ Note that while the physically relevant boundary for the Earth's fluid core is a spherical annulus, for the purposes of studying the mathematical properties of the $M G$ equations we simply consider periodic boundary conditions.
} 
for $k=\left(k_{1}, k_{2}, k_{3}\right) \in \mathbb{Z}_{*}^{3}:=\mathbb{Z}^{3} \backslash\left\{k_{3}=0\right\}$. On $\left\{k_{3}=0\right\}$ we define $\hat{M}_{\nu j}(k)=0$ for all $j \in\{1,2,3\}$, since $\theta$ and $u$ have zero vertical mean.

In summary, for $\nu, \kappa \geq 0$, the magnetogeostrophic $\mathrm{MG}_{\nu, \kappa}$ equation is the nonlinear advection diffusion equation (37), in which the incompressible velocity field $u=M_{\nu}[\theta]$ is given by the constitutive law (41). Some of the key properties of the $M G_{\nu, \kappa}$ equation are that it is three dimensional, its diffusion is given by the classical Laplacian (when $\kappa>0$ ), and the symbol $\hat{M}_{\nu}(k)$ is orthogonal to the wavevector $k$ and is an even function of it. Most importantly, the nature of the operator $M_{\nu}$ changes dramatically between the inviscid case $\nu=0$ (when $M_{\nu}$ is an unbounded operator) and the dissipative case $v>0$ (when $M_{\nu}$ is a smoothing operator). This latter fact plays a crucial role in the analysis of $\mathrm{MG}_{\nu, \kappa}$.

It is instructive to compare the $\mathrm{MG}_{\nu, \kappa}$ equation to more classical hydrodynamic models in the canon of nonlinear active scalar equations, such as the $2 \mathrm{D}$ surface quasigeostrophic equation (SQG) and its dissipative versions. The SQG equation has received tremendous interest in the mathematical community over the past decades, through works of Constantin, Wu, Cordoba, Caffarelli-Vasseur, Kiselev-Nazarov-Volberg, and many many others. The recent results in the analysis of the SQG equation have played a fundamental role in developing the mathematical foundations for the $\mathrm{MG}_{\nu, \kappa}$ model.

4.2. The inviscid model $v=0$. In Friedlander's original work on this subject [FV11], it is shown that when $v=0$ there exist regions of Fourier space where $\hat{M}_{0}(k)$ is unbounded and may even grow as fast as $|k|$ when $|k| \rightarrow \infty$. Thus, in this worst-case scenario $M_{0}$ acts as an order one Fourier multiplier, and so the map $\theta \mapsto u=M[\theta]$ effectively loses one derivative. Since the evolution of $\theta$ in (37) satisfies the maximum principle (when $S=0$ ) one may prove an a priori bound on $\theta \in L_{t}^{\infty} L_{x}^{\infty}$; this is the strongest norm of $\theta$ which is a priori bounded uniformly in time. In turn, in view of the aforementioned properties of $M_{0}$, we may only deduce a bound on $u$ in $L_{t}^{\infty} B M O_{x}^{-1}$, i.e., $u$ behaves as the divergence of a $B M O$ skew-symmetric matrix. The crucial observation is that in three dimensions advection-diffusion equations with incompressible $L_{t}^{\infty} B M O_{x}^{-1}$ drifts are critical, meaning that the natural parabolic scaling of equations leaves the size of the rescaled drift velocity $u$ unchanged in this norm. When the initial datum $\theta_{0}$ or the forcing term $S$ are large, it is notoriously difficult to establish the global existence of smooth solutions for such problems, and indeed, prior to [FV11] this was an open problem. Using a variant of the parabolic De Giorgi iteration and the incompressibility of $u$, Friedlander and the last author have proven the following.
Theorem 4.1 ([FV11]). Let the initial data $\theta_{0} \in L^{2} \cap L^{\infty}$ and $\theta \in L^{\infty}\left(0, \infty ; L^{2}\right) \cap L^{2}\left(0, \infty ; H^{1}\right)$ be a weak solution of $M G_{0, \kappa^{\prime}}$ where $\kappa>0$. Then, for any $t_{0}$, there exists $\alpha>0$ such that $\theta \in C^{\alpha / 2}\left(t_{0}, \infty ; C^{\alpha}\right)$.

The proof of Theorem 4.1 starts by using De Giorgi iteration to establish the boundedness of the weak solutions to $\mathrm{MG}_{0, \kappa}$. Proving Hölder regularity requires a more delicate argument since the drift velocity $u$ is the divergence of a $B M O$, rather than $L^{\infty}$, matrix. By essentially using the divergence-free nature of $u$, and by appealing to the John-Nirenberg inequality, one may however prove a suitable $L^{p}$-based Caccioppoli inequality, which is the key ingredient in De Giorgi's improvement of the oscillation lemma. Note that while Theorem 4.1 only establishes global Hölder continuity of weak solutions to $\mathrm{MG}_{0, \kappa}$ a posteriori one may deduce these solutions are $C^{\infty}$ smooth for $t>0$, and thus unique.

Having established the global existence of smooth solutions for $\mathrm{MG}_{0, \kappa}$ with $\kappa>0$, Friedlander and her collaborators have turned their attention to proving the existence of instabilities for the nonlinear model, since this is after all what the geodynamo problem asks for. Building on the ideas discussed in Section 2, Friedlander was able to prove that for $m \geq 1$, the operator corresponding to $M_{0, \kappa}$ linearized around the steady state $\Theta_{0}=\sin \left(m x_{3}\right)$, with associated $U_{0}=0$ and forcing $S=\mathrm{km}^{2} \sin \left(m x_{3}\right)$, has unstable point spectrum. Moreover, the largest eigenvalue has real part which is at least as large as $2^{-9} \mathcal{K}^{-1}$ once $\kappa$ is taken to be sufficiently small and $m \ll \kappa^{-1}$. As in Section 2, a careful analysis shows that this linear instability implies that solutions to the full nonlinear $M_{0, \kappa}$ are Lyapunov nonlinearly unstable: initially small perturbations of $\Theta_{0}$ grow exponentially in time, which is consistent with the dynamo instabilities.

Friedlander, jointly with Rusin and the last author, has obtained a number of further results concerning the $M_{0, \kappa}$ model. For instance, the system $\mathrm{MG}_{0,0}$ is Hadamard illposed in Sobolev spaces, but local well-posedness is recovered if one adds back a dissipative operator of the type $\kappa(-\Delta)^{\gamma}$ with $\gamma \geq 1 / 2$. For further results in the inviscid case, we refer the interested reader to the review paper [FRV14]. 4.3. The viscous model $\nu>0$. The nature of the $M G_{v, k}$ equations changes dramatically when considering the viscous case $\nu>0$. To see this, return to the symbol $\hat{M}_{\nu}$ defined implicitly by (41). For $\nu>0$, instead of having an unbounded symbol, one may show that $|k|^{2}\left|\hat{M}_{\nu}(k)\right| \lesssim_{\nu} 1$ for all $k \in \mathbb{Z}^{3}$. Thus, the map $\theta \mapsto u=M[\theta]$ is smoothing of order two in the viscous case; a regularization that is even stronger than the Biot-Savart law. Thus, an a priori estimate on $\theta \in L_{t}^{\infty} L_{x}^{\infty}$ (natural in view of the maximum principle for (36)), yields a bound for $\nabla^{2} u$ in $L_{t}^{\infty} L_{x}^{p}$ for any $p<\infty$. In particular, the Lipschitz norm of $u$ is a priori 
controlled, globally in time, and as for classical ODEs, one may thus hope that the system is globally well-posed even when the diffusivity parameter $\kappa$ vanishes. This problem was recently resolved by Friedlander jointly with Suen.

Theorem 4.2 ([FS15]). Consider $v>0$ and $\kappa \geq 0$. Assume that $\theta_{0} \in L^{3}$ has zero mean. Then, there exists a unique global weak solution $\theta \in B C\left((0, \infty) ; L^{3}\right)$ with $u \in C\left((0, \infty) ; W^{2,3}\right)$ of the $M G_{\nu, \kappa}$ equation.

The remarkable fact about the above result is that it holds even for $\kappa=0$. It is also shown in [FS15] that solutions to $\mathrm{MG}_{\nu, \kappa}$ converge in the vanishing viscosity limit $\nu \rightarrow 0$ towards solutions of $\mathrm{MG}_{0, \kappa}$ for $\kappa>0$. Moreover, there is no anomalous dissipation of energy for (37) in the vanishing diffusivity limit: for any $T>0$ and $\theta_{0} \in H^{1}$, we have that

$$
\lim _{\kappa \rightarrow 0} \int_{0}^{T} \int_{\mathbb{T}^{3}} \kappa\left|\nabla \theta_{\kappa, \nu}\right|^{2} \mathrm{~d} x \mathrm{~d} t=0,
$$

where $\theta_{\kappa, \nu}$ denotes the unique solution of $\mathrm{MG}_{\nu, \kappa}$ guaranteed by Theorem 4.2. The above result addresses the question of magnetogeostropic turbulence raised by Moffatt and Loper [ML94]. Concerning the geodynamo problem, using techniques similar to those in Section 2, it was proven by Friedlander and Suen in [FS15] that the dissipative $\mathrm{MG}_{\nu, \kappa}$ equation sustains exponentially growing dynamo-type instabilities.

4.4. Singular limits for the magnetogeodynamo in a stochastic setting. Another direction of Friedlander's work in magnetohydrodynamics concerns the Moffatt-Loper model (34)-(37) in the stochastic setting, where the source term $S$ in thermal evolution equation (37) is specified as a Gaussian white noise. This probabilistic setting of the model interprets white noise driven terms as a heat source which "continuously regenerates the statistically stationary temperature distribution throughout the core" as described by Moffat and Loper [ML94]. As such, an important feature of Friedlander and her collaborators' work in the stochastic setting is to analyze statistically invariant states, i.e., to study invariant measures of the associated Markovian dynamics. More broadly, such measures play an important role in the study of turbulence as they provide a framework for identifying robust statistical quantities in turbulent flows.

In a joint work with Földes, the second coauthor, and Richards [FFGHR17], Friedlander considered the singular parameter limits $R_{o}, R_{m} \rightarrow 0$ for (34)-(35), with these limits being carried out in terms of the corresponding invariant measures. Roughly speaking, the work [FFGHR17] establishes that statistically robust quantities of the full MHD system with $0<R_{o}, R_{m} \ll 1$ are well approximated by those measured using the formal limit system $R_{o}=R_{m}=$ 0 . More precisely, we summarize this result as follows.
Theorem 4.3 ([FFGHR17]). Consider (34)-(37) with $\nu, \kappa>$ 0 , in the presence of a stochastic source term of the form

$$
S(x, t)=\sum_{\substack{k \in \mathbb{Z}_{0}^{3} \\ m \in\{0,1\}}} \alpha_{k, m} \sigma_{k}^{m}(x) \dot{W}^{k, m}(t),
$$

where $\sigma_{k}^{0}(x):=\cos (k \cdot x), \sigma_{k}^{1}(x):=\sin (k \cdot x), \alpha_{k} \in \mathbb{R}$ are amplitudes, and $\left\{\dot{W}^{k, m}\right\}$ is a collection of independent white noise processes. Subject to the nondegeneracy (hypoellipticity) condition that $\alpha_{(1,0,0) m}, \alpha_{(0,1,0) m}, \alpha_{(0,0,1) m}$ are nonzero for $m=0,1$ the limit equation (34)-(37) when $R_{o}=R_{m}=0$ has a unique statistically invariant state $\mu$ which is achieved at an exponential rate; $c f .(44)$ below. For any collection of statistically invariant states $\left\{\mu_{R_{o}, R_{m}}\right\}_{R_{o}, R_{m}>0}$ and any suitably regular observable $\Phi$ of the dynamics, we have

$$
\begin{aligned}
\mid \int \Phi(u, b, \theta) \mathrm{d} \mu_{R_{o}, R_{m}}-\int \Phi( & u, b, \theta) \mathrm{d} \mu \mid \\
& \leq C\left(R_{o}+R_{m}\right)^{\gamma},
\end{aligned}
$$

where the constants $\gamma, C>0$ are independent of $R_{o}, R_{m}>0$.

An interesting feature of the above result is that the estimate (43) is independent of possible nonuniqueness in the approximating statistics. Here we observe that the formal limit system when $R_{o}=R_{m}=0$, i.e., $\mathrm{MG}_{\nu, \kappa^{\prime}}$ is an active scalar equation with a smoothing constitutive law of order two, and therefore classically yields a Markovian dynamic. On the other hand, for positive values of $R_{o}, R_{m}$ it is not clear that (34)-(37) is well posed or that the associated statistically $\mu_{R_{o}, R_{m}}$ steady states are unique; for positive $R_{o}, R_{m}$, the invariant states $\mu_{R_{o}, R_{m}}$ are considered as stochastic analogues (i.e., martingale solutions) of stationary Leray weak solutions.

The strategy in [FFGHR17] turns on establishing a spectral gap condition is suitable in a Wasserstein metric for the Markovian dynamics for the limit system. Here, one considers

$$
\mathfrak{W}_{\rho}(\mu, \nu):=\inf _{\Gamma \in \mathcal{C}_{\mu, \nu}} \int \rho(U, V) \Gamma(\mathrm{d} U, \mathrm{~d} V),
$$

where $\rho$ is taken to be a certain metric, topologically equivalent to $L^{2}$, but which punishes elements far from the origin, and $\mathcal{C}_{\mu, \nu}$ is the set of couplings of $\mu$ and $\nu$. "Weak Harris" mixing results for Markovian systems which are adapted to such topologies $\mathfrak{W}_{\rho}$ were developed by Hairer, Mattingly, Kuksin, Shirikyan, and others in the early 2000s. By leveraging such modern variants of Harris' classical theorems one may establish bounds of the type

$$
\mathfrak{W}_{\rho}\left(\mu P_{t}, \nu P_{t}\right) \leq C e^{-\kappa t} \mathfrak{W}_{\rho}(\mu, \nu) .
$$

A crucial step in the analysis leading to (44) is to study the delicate interactions of the stochastic source terms $S$ in (42) with the nonlinear portion of the drift $u \cdot \nabla \theta$ present in (41), in order to establish a certain Hörmander-type hypoellipticity condition. This analysis produces a form of 
smoothing in the Markovian dynamics, the so-called asymptotic strong Feller condition of Hairer and Mattingly.

The bound (44) crucially affords a reduction of the study of the convergence of the stationary states in (43), to the establishing of bounds between positive and limit solutions in the parameters $R_{o}, R_{m}$ at a fixed finite time $t_{*}>0$. The finite time convergence analysis produces interesting challenges due to a phase space mismatch between the full system when $R_{o}, R_{m}>0$ and the active scalar equation representing the limit. This formally suggests a multitime scale analysis to correctly approximate solutions at an initial layer in time. One insight in [FFGHR17] is that, beyond the initial layer, there is no need to correct the dynamics in order to obtain a bound similar to (43) for the thermal components of the dynamics; these decay rates may then be transferred to bounds on velocity and magnetic components.

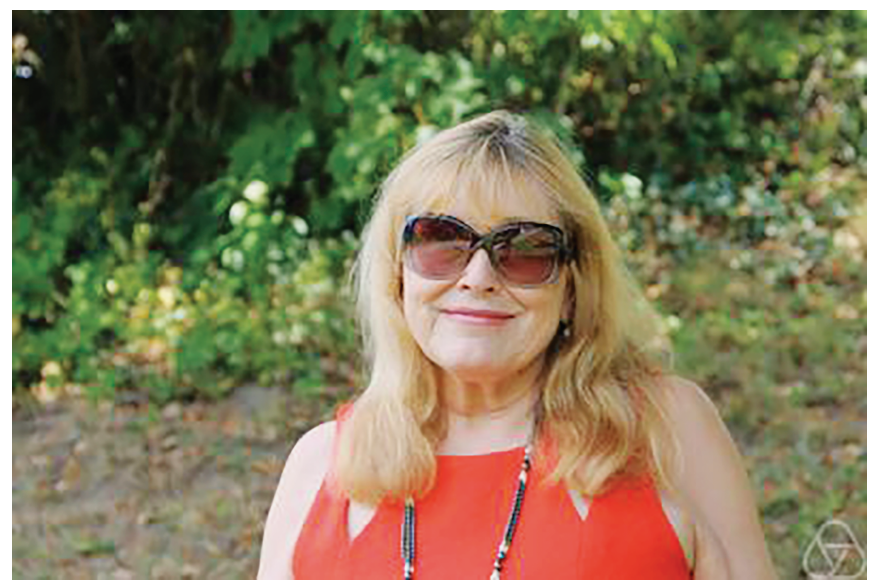

Figure 1. Friedlander at the Mathematisches Forschungsinstitut Oberwolfach in 2015.

\section{Impact of Friedlander's Work}

It is hard to overestimate the significance of Friedlander's research and her impact on the work of so many mathematicians, including the authors of this survey. The geometric optics method has developed into a powerful tool to study instabilities for a broad range of fluid models. Further advances in this area have led to a full description of the essential spectrum of the 2D and 3D Euler system. This, in turn, made it possible to apply methods from dynamical systems to construct invariant manifolds near unstable equilibria.

Friedlander's results on the long-time behavior of dyadic models represent a prototype of the "dream scenario" for the full Euler equations-finite time blow-up or regularization that leads to the Onsager-critical regularity for solutions with any initial data. The regularization property of the nonlinear term has been investigated further starting with the work of Barbato, Morandin, and Romito.
A recent work of Tao exploits the blow-up mechanism of the dyadic model and adds the energy cascade delays to break the 4D barrier that result in a construction of an "averaged" 3D Navier-Stokes model which fulfills all the same energy estimates as the actual Navier-Stokes, but blows up in finite time-a long-awaited result demonstrating that the energy method alone is not enough to resolve the Clay Problem for 3D Navier-Stokes.

The MG model has sparked research in many directions, and in particular it was one of the first active scalar equations to which the convex integration method was adapted. It played a crucial step in understanding the role of symmetries in the Fourier multiplier for the scalar-to-velocity constitutive law, as a mechanism for generating wild solutions.

Friedlander's contribution to the ongoing work on the Onsager conjecture laid the basis for the mathematical formalization of the physical concepts of intermittency and accumulation set for the forward energy cascade in turbulent flows. These concepts proved to be experimentally measurable and were well received in the physical community. Friedlander's analytical results on the energy law remain sharp to date and were extended to fluids models with boundaries, to inhomogeneous and compressible fluids, and even to general hyperbolic conservation laws.

\section{References}

[CCFS08] A. Cheskidov, P. Constantin, S. Friedlander, and R. Shvydkoy, Energy conservation and Onsager's conjecture for the Euler equations, Nonlinearity 21 (2008), no. 6, 12331252, DOI 10.1088/0951-7715/21/6/005. MR2422377

[CF09] Alexey Cheskidov and Susan Friedlander, The vanishing viscosity limit for a dyadic model, Phys. D 238 (2009), no. 8, 783-787, DOI 10.1016/j.physd.2009.01.011 MR2522972

[CFP07] Alexey Cheskidov, Susan Friedlander, and Nataša Pavlović, Inviscid dyadic model of turbulence: the fixed point and Onsager's conjecture, J. Math. Phys. 48 (2007), no. 6, 065503, 16, DOI 10.1063/1.2395917. MR2337019

[CFP10] Alexey Cheskidov, Susan Friedlander, and Nataša Pavlović, An inviscid dyadic model of turbulence: the global attractor, Discrete Contin. Dyn. Syst. 26 (2010), no. 3, 781794, DOI 10.3934/dcds.2010.26.781. MR2600714

[Che08] Alexey Cheskidov, Blow-up in finite time for the dyadic model of the Navier-Stokes equations, Trans. Amer. Math. Soc. 360 (2008), no. 10, 5101-5120, DOI 10.1090/S0002-994708-04494-2. MR2415066

[CSF12] Alexey Cheskidov, Roman Shvydkoy, and Susan Friedlander, A continuous model for turbulent energy cascade, Mathematical aspects of fluid mechanics, London Math. Soc. Lecture Note Ser., vol. 402, Cambridge Univ. Press, Cambridge, 2012, pp. 52-69. MR3050291

[FFGHR17] Juraj Földes, Susan Friedlander, Nathan GlattHoltz, and Geordie Richards, Asymptotic analysis for randomly forced MHD, SIAM J. Math. Anal. 49 (2017), no. 6, 4440-4469, DOI 10.1137/16M1071857. MR3723323 
[FP04] Susan Friedlander and Nataša Pavlović, Blowup in a three-dimensional vector model for the Euler equations, Comm. Pure Appl. Math. 57 (2004), no. 6, 705-725, DOI 10.1002/cpa.20017. MR2038114

[FRV14] S. Friedlander, W. Rusin, and V. Vicol, The magnetogeostrophic equations: a survey, Proceedings of the St. Petersburg Mathematical Society. Vol. XV. Advances in mathematical analysis of partial differential equations, Amer. Math. Soc. Transl. Ser. 2, vol. 232, Amer. Math. Soc., Providence, RI, 2014, pp. 53-78, DOI 10.1090/trans2/232/04. MR3287785

[FS05] Susan Friedlander and Roman Shvydkoy, The unstable spectrum of the surface quasi-geostropic equation, J. Math. Fluid Mech. 7 (2005), no. suppl. 1, S81-S93, DOI 10.1007/s00021-004-0129-3. MR2126131

[FS15] Susan Friedlander and Anthony Suen, Existence, uniqueness, regularity and instability results for the viscous magneto-geostrophic equation, Nonlinearity 28 (2015), no. 9, 3193-3217, DOI 10.1088/0951-7715/28/9/3193. MR3403395

[FSV97] Susan Friedlander, Walter Strauss, and Misha Vishik, Nonlinear instability in an ideal fluid (English, with English and French summaries), Ann. Inst. H. Poincaré Anal. Non Linéaire 14 (1997), no. 2, 187-209, DOI 10.1016/S0294. 1449(97)80144-8. MR1441392

[FV11] Susan Friedlander and Vlad Vicol, Global wellposedness for an advection-diffusion equation arising in magneto-geostrophic dynamics, Ann. Inst. H. Poincaré Anal. Non Linéaire 28 (2011), no. 2, 283-301, DOI 10.1016/j.anihpc.2011.01.002. MR2784072

[FV91] Susan Friedlander and Misha M. Vishik, Dynamo theory, vorticity generation, and exponential stretching, Chaos 1 (1991), no. 2, 198-205, DOI 10.1063/1.165829. MR1135909

[FV92] Susan Friedlander and Misha M. Vishik, Instability criteria for steady flows of a perfect fluid, Chaos 2 (1992), no. 3, 455-460, DOI 10.1063/1.165888. MR1184488

[KP02] Nets Hawk Katz and Nataša Pavlović, A cheap Caffarelli-Kohn-Nirenberg inequality for the Navier-Stokes equation with hyper-dissipation, Geom. Funct. Anal. 12 (2002), no. 2, 355-379. MR1911664

[KP05] Nets Hawk Katz and Nataša Pavlović, Finite time blowup for a dyadic model of the Euler equations, Trans. Amer. Math. Soc. 357 (2005), no. 2, 695-708. MR2095627

[ML94] Henry Keith Moffatt and David Loper, The magnetostrophic rise of a buoyant parcel in the earth's core, Geophysical Journal International 117 (1994), no. 2, 394-402.

[VF03] Misha Vishik and Susan Friedlander, Nonlinear instability in two dimensional ideal fluids: the case of a dominant eigenvalue, Comm. Math. Phys. 243 (2003), no. 2, 261273, DOI 10.1007/s00220-003-0963-9. MR2021907

[VF93] Misha M. Vishik and Susan Friedlander, Dynamo theory methods for hydrodynamic stability, J. Math. Pures Appl. (9) 72 (1993), no. 2, 145-180. MR1216094

[Vis89] M. M. Vishik, Magnetic field generation by the motion of a highly conducting fluid, Geophys. Astrophys. Fluid Dynam. 48 (1989), no. 1-3, 151-167, DOI 10.1080/03091928908219531. MR1024693

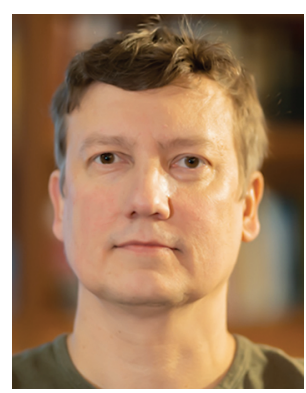

Alexey Cheskidov

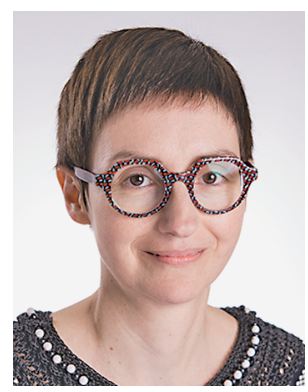

Natasa Pavlovic

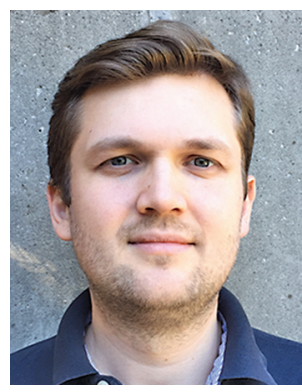

Vlad Vicol

\section{Credits}

Opening photo is courtesy of Nedyu Popivanov/Fields Institute.

Figure 1 is courtesy of the Mathematisches Forschungsinstitut Oberwolfach.

Photo of Alexey Cheskidov is courtesy of Alexey Cheskidov.

Photo of Nathan Glatt-Holtz is courtesy of Nathan GlattHoltz.

Photo of Natasa Pavlovic is courtesy of the University of Texas at Austin.

Photo of Roman Shvydkoy is courtesy of Roman Shvydkoy.

Photo of Vlad Vicol is courtesy of Simona Vicol. 